\title{
Interactive comment on "A dataset of tracer concentrations and meteorological observations from the Bolzano Tracer EXperiment (BTEX) to characterize pollutant dispersion processes in an Alpine valley" by Marco Falocchi et al.
}

\section{Anonymous Referee \#1}

Received and published: 9 September 2019

The paper describes an experimental campaign carried out in an alpine valley. The measurements include both meteorological and concentration data. The measurement techniques and strategy are shown in details as well as the gathered data-sets. Observations of meteorological quantities and tracer concentrations are very useful for both understanding the flow and turbulence dynamics and evaluating the performances of the numerical models, especially in complex terrain. They are particularly interesting and useful when available for all the scientific community. For these reasons a strongly support the publication of the paper. I have only minor comments and suggestions for 
the authors.

Line 39: TRACT experiments might be cited here.

Lines 111-115: It is not clear whether two different kinds of system were used and two different systems to fill them were adopted.

Interactive

Line 123: I wonder if "predicted" would be better than "foreseen"

comment

Line 130: NWP simulations at a resolution of $200 \mathrm{~m}$ are not standard. This resolution, although justified by the need to describe small scales in the complex terrain, falls in the "grey zone" of the atmosphere. Thus, this point deserves some discussion.

Table 2: It may be useful to write which kind of instrument the information given in the table refer to (not only the technical name)

Section 3.2: I wonder whether, for reasons of clarity, the description of the instrumentation for measuring the SF6 concentrations, bottles and bags, might be moved here.

Line 202: Add MTP to indicate the instrument utilised for the measurements.

Line 213: Please, indicate which kind of data are used to plot the geopotential height map.

Line 269: Substitute Figure with Fig. as for the others.

Figure 8: I have some concerns about this figure. It does not show the positions of the sampling points along the west-est direction. Further, since some of the samplings last less than others, it seems that the concentration would be zero at some time which, instead, might be not true.

Line 277: The text refers to Bolzano which is not shown on the maps.

Line 285: It seems that concentrations were found upwind to the incinerator.

Conclusions: Nothing is said about the strategy adopted to locate the samplings. Did it succeed? Did the model correctly predict the plume dispersion helping to properly 
positioning bottles and bags? Were numerical simulations repeated and the results compared with the observations?

ESSDD

Line 309: Were simulations done and compared with the measured data?

Interactive comment on Earth Syst. Sci. Data Discuss., https://doi.org/10.5194/essd-2019-120, 2019.

Interactive

comment 